\title{
Treatment of Vascular Thrombosis in Antiphospholipid Syndrome: An Update
}

\author{
Lida Kalmanti ${ }^{1}$ Edelgard Lindhoff-Last ${ }^{1}$ \\ ${ }^{1}$ Coagulation Center and Cardiology-Angiology Center Bethanien \\ Hospital (CCB), Frankfurt, Germany \\ Hämostaseologie 2020;40:31-37.
}

Address for correspondence Edelgard Lindhoff-Last, MD, Cardiology Angiology Center Bethanien Hospital (CCB), Im Prüfling 23, 60389

Frankfurt, Germany (e-mail: e.lindhoff-last@ccb.de).

\author{
Abstract \\ Keywords \\ - antiphospholipid \\ syndrome \\ - arterial thrombosis \\ - venous thrombosis \\ - vitamin $\mathrm{K}$ antagonists \\ - direct oral \\ anticoagulants
}

The antiphospholipid syndrome (APS) is an acquired autoimmune disorder associated with arterial, venous, or microvascular thrombosis and/or pregnancy complications mainly in young age. The diagnosis is made by the persistent detection of anticardiolipin antibodies, B2-glycoprotein I antibodies (B2GPIA), and/or lupus anticoagulants (LAs) for at least 12 weeks. Patients should present with at least one clinical and one laboratory criterion. Patients presenting with all three types of antibodies and vascular events are high-risk patients and should receive vitamin $\mathrm{K}$ antagonists (VKAs) as long as the antibodies persist. In patients with prior arterial thrombosis, VKA with or without low-dose aspirin is the current treatment of choice. The international normalized ratio (INR) should be between 2 and 3 although in some cases keeping the target INR above 3 may be necessary. Patients with venous thrombosis and negative LA may alternatively be treated with direct oral anticoagulants although more data are needed. Minimizing vascular risk factors is always necessary in APS patients. Aspirin can be given as primary prevention in asymptomatic patients with positive antiphospholipid antibodies without thrombosis or pregnancy complications especially when additional vascular risk factors are present. Catastrophic APS occurs in less than $1 \%$ of APS patients and presents as a thrombotic storm. Early use of a combined triple therapy such as anticoagulation, plasma exchange, and steroids with either or not addition of immunoglobulins is important to reduce mortality.

\section{Introduction}

The antiphospholipid syndrome (APS) is an acquired systemic autoimmune disorder associated with arterial, venous, or microvascular thrombosis, and/or pregnancy complications in young age with $85 \%$ of patients being between 15 and 50 years of age. ${ }^{1}$

The diagnosis is based upon the revised Sapporo classification criteria which include persistent detection of anticardiolipin antibodies (ACAs), $\beta 2$-glycoprotein I antibodies ( $\beta 2$ GPIA), and/or lupus anticoagulants (LAs) for at least 12 weeks. Patients should present with at least one clinical and one laboratory criterion. 2,3

Primary antiphospholipid syndrome (PAPS) is characterized by the presence of one or more types of antiphospho-

received

September 4, 2019

accepted after revision

November 27, 2019

lipid antibodies (APLs) without an underlying disease. In secondary APS (SAPS) an underlying autoimmune disorder, e.g., systemic lupus erythematosus (SLE) or other conditions, such as malignancy or infection, is present.

Catastrophic APS (CAPS) is a rare, severe disease with high mortality characterized by the presence of thrombosis in multiple organs. ${ }^{4}$

Furthermore, APS can present with cardiac manifestations, most frequently heart valve thickening affecting the mitral or aortic valve and early onset atherosclerosis. ${ }^{5}$

\section{Laboratory Diagnosis}

ACA antibody type immunoglobulin G ( $\operatorname{IgG}$ ) or immunoglobulin $M(\operatorname{IgM})$ and $32 \mathrm{GPIA}$ type IgG or IgM are measured by

(C) 2020 Georg Thieme Verlag KG Stuttgart · New York
DOI https://doi.org/ 10.1055/s-0040-1701473. ISSN 0720-9355. 
enzyme-linked-immunosorbent serologic assay (ELISA)-based assays and titers higher than the 99th percentile have been correlated with clinical events. Detection of LAs is based on phospholipid-dependent clotting assays. Antibody positivity should be confirmed on two or more occasions with a distance of 12 weeks. A single measurement should not be considered as positive as various conditions such as infections can cause a transient increase of APL or LA. For all tests, the 99th percentile should be used as the cut-off for the determination of the reference ranges.

After confirmation of the diagnosis, regular monitoring of APL should be performed every 6 to 12 months, since APLtiter might decrease over time and continuous anticoagulation is only needed as long as APLs are present.

It is important to notice that oral anticoagulants such as vitamin K antagonists (VKAs) and direct oral anticoagulants (DOACs) can falsify the results of LAs and may lead to falsely positive assay results. Therefore, for correct laboratory diagnosis the patient should be switched to low-molecularweight heparin or should be measured after the end of the oral anticoagulant treatment (after five half-life times). Alternatively, in vitro use of special absorbers (i.e., DOACStop) can reverse the effects of DOACs on LA testing. ${ }^{6}$

\section{Risk Profiles According to the Presence of APL}

According to the type and levels of APLs, patients are divided into high, moderate, or low thrombotic risk (see - Table 1). High-risk patients are those with triple-positive APL (LA, ACA, and, $\beta 2$ GPIA). ${ }^{7-9}$ Patients presenting with LA with or without ACA and/or $\beta 2$ GPIA frequently also exhibit a higher rate of thrombotic events and higher mortality rates. ${ }^{10,11}$ Therefore, these patients should also be considered as high-risk patients. Moderate-risk patients are those with negative LA and a moderate-to-high titer of ACA and B2GPIA (double positivity) or a moderate-to-high titer of ACA or 2GPIA alone (single positivity). ${ }^{1,8}$ Low-risk patients are those patients with negative LA and a low titer of ACA and/or B2GPIA.

A global APS score (GAPSS) has been developed in a single center to better stratify patients. This score consists of the

Table 1 Risk assessment according to the antiphospholipid antibody (APL) profile of patients with the antiphospholipid syndrome modified according to Garcia and Erkan ${ }^{7}$

\begin{tabular}{|l|l|}
\hline Risk profile & Laboratory findings \\
\hline High risk & $\begin{array}{l}\text { - Triple positive APL (LA, ACA, and B2GPIA) } \\
\text { - Positive LA with or without ACA or } \beta 2 \text { GPIA } \\
\text { (single or double positivity with LA) }\end{array}$ \\
\hline $\begin{array}{l}\text { Moderate } \\
\text { risk }\end{array}$ & $\begin{array}{l}\text { Negative LA and moderate to high titer of } \\
\text { ACA and/or } \beta 2 \text { GPIA } \\
\text { (single or double positivity without LA) }\end{array}$ \\
\hline Low risk & $\begin{array}{l}\text { Negative LA and low titer of ACA and/or } \\
\text { B2GPIA (single or double positivity without LA) }\end{array}$ \\
\hline
\end{tabular}

Abbreviations: $\beta 2$ GPIA, $\beta 2$-glycoprotein I antibodies; APL, antiphospholipid antibodies; ACA, anticardiolipin antibodies; LA, lupus anticoagulant. presence of four different antibody types and two conventional cardiovascular risk factors: hyperlipidemia and arterial hypertension. As anti-phosphatidylserine (anti-PS) and prothrombin (PT) antibodies are not routinely used in most centers but are included in the GAPSS score, an adjusted GAPSS (aGAPSS) score has been proposed. The aGAPSS score excludes these antibodies and is currently being evaluated.

Recently the APS alliance for clinical trials and international networking (APS ACTION) retrospectively analyzed 379 patients with thrombotic APS. ${ }^{12}$ A total of $68 \%$ of the patients had primary APS. The authors observed a higher aGAPSS in patients with recurrent thrombosis, either arterial or venous. As this is a retrospective study, no available information about the different laboratory methods and no information about the cardiovascular risk factors at the time of recurrence of thrombosis is provided.

Therefore, prospective international studies are needed to gain more information about the utility of this score to detect patients at higher risks who might need a more intensive therapy.

\section{Primary Prevention in Asymptomatic Patients with Positive APL}

Given the low background risk of thrombosis in the general population, the absolute risk of a first thrombosis in APLpositive patients who do not have other risk factors is probably less than $1 \%$ per year. As in the general population, arterial and venous thrombotic events in APL-positive patients are often multicausal.In contrast, the annual risk of a first thrombosis in patients with persistently moderate-to-high risk APL profiles and a systemic autoimmune disease or additional thrombotic risk factors may be as high as $5 \% .^{5}$ The high thrombotic risk of triple-APL-positive patients is also observed in asymptomatic individuals, in whom the risk of thrombosis is significantly higher than in those with single-APL positivity. ${ }^{13}$

The use of low-dose aspirin for primary thrombosis prevention is still controversial, given the low quality of evidence and lack of prospective data documenting that this strategy is effective. ${ }^{5}$

For asymptomatic patients with positive APLs without clinical symptoms, prophylactic low-dose aspirin is therefore only recommended in patients with moderate-to-high risk APL profiles and a systemic autoimmune disease or additional vascular risk factors (see - Table 2). ${ }^{11}$ In addition, thrombosis prophylaxis with low-molecular-weight heparin or DOAC may be considered in risk situations (i.e., long-distance flights, immobilization, etc.) to prevent venous thrombosis.

\section{Secondary Prevention of Venous Thromboembolism in Patients with APS}

In patients presenting with venous thrombosis, APS is observed in up to $10 \%$ of these patients. Venous thromboembolism (VTE) can present as deep vein thrombosis, pulmonary embolism (PE), superficial thrombophlebitis, or less frequently as thrombosis at unusual sites (e.g., cerebral venous thrombosis, portal vein thrombosis, mesenterial vein thrombosis). 
Table 2 Primary thromboprophylaxis in APL-positive subjects without clinical symptoms of the antiphospholipid syndrome

\begin{tabular}{|l|l|l|}
\hline Risk profile & Treatment & Additional therapy \\
\hline $\begin{array}{l}\text { Asymptomatic patients with } \\
\text { high or moderate risk APL } \\
\text { profile }\end{array}$ & $\begin{array}{l}\text { Low-dose aspirin }(75-100 \mathrm{mg}) \text {. Consider thromboprophylaxis with } \\
\text { NMH or DOAC in risk situations (i.e., long-distance flights, immobili- } \\
\text { zation, operation, etc.) }\end{array}$ & $\begin{array}{l}\text { Minimize vascular } \\
\text { risk factors }^{\text {a }}\end{array}$ \\
\hline $\begin{array}{l}\text { Asymptomatic patients with } \\
\text { low risk APL profile }\end{array}$ & Low-dose aspirin $(75-100 \mathrm{mg})$ may or may not be considered & $\begin{array}{l}\text { Minimize vascular } \\
\text { risk factors }^{\text {a }}\end{array}$ \\
\hline $\begin{array}{l}\text { Asymptomatic SLE patients } \\
\text { with positive APL profile }\end{array}$ & $\begin{array}{l}\text { Low-dose aspirin }(75-100 \mathrm{mg}) \text { and hydroxychloroquine. } \\
\text { Consider thromboprophylaxis with NMH or DOAC in risk situations (i.e., } \\
\text { long-distance flight, immobilization, operation, etc.) }\end{array}$ & $\begin{array}{l}\text { Minimize vascular } \\
\text { risk factors }^{\text {a }}\end{array}$ \\
\hline
\end{tabular}

Abbreviations: APL, antiphospholipid antibodies; DOAC, direct oral anticoagulant; NMH, low molecular weight heparin; SLE, systemic lupus erythematosus.

${ }^{\text {a }}$ Smoking cessation, weight loss in obese patients, control of arterial hypertension, hyperlipidemia, and diabetes mellitus.

The majority of APS patients develop VTE (up to 70\%). ${ }^{1,5}$

The mainstay treatment of patients with triple-positive APL and venous thrombosis is VKAs (see - Table 3).

Data from studies with patients experiencing venous events showed no benefit of a target international normalized ratio (INR) of 3 to 4 in comparison to an INR of 2 to $3 .^{11,12}$ However, monitoring anticoagulation with VKA in patients with APS may be challenging as some PT assays are sensitive to the effects of LA. This can cause an overestimation of the INR. In this case we recommend measuring the factor $X$ activity by the use of a chromogenic factor X-assay as the results are not influenced by the presence of APL. ${ }^{14}$

Patients with unprovoked thrombosis and persistent APL should receive long-term anticoagulation ${ }^{14,15}$ after their first venous thrombosis as a high recurrence rate of thrombosis is observed after stopping anticoagulation. ${ }^{16}$ Approximately $10 \%$ of APS patients overall ${ }^{2}$ and $30 \%$ of those who are triple-APL positive ${ }^{12}$ have recurrent thrombotic events, arterial or venous, while receiving VKAs (at standard intensity, target INR of 2.5 ) at 5 -year follow-up. ${ }^{13}$ The addition of aspirin in patients with APS and VTE is not required. ${ }^{1}$

So far little is known about the efficacy and safety of DOACs in APS patients with venous thrombosis. The prospective randomized, controlled, open-label, noninferiority RAPS trial (rivaroxaban vs. warfarin to treat patients with thrombotic APS with or without SLE) reported no thrombotic or major bleeding events among 116 APS patients with venous thrombosis who were randomly allocated to receive rivaroxaban versus warfarin for 210 days. ${ }^{15} \mathrm{~A}$ limitation of RAPS is that it was not designed to confirm clinical efficacy and long-term safety. Rather, the trial was designed with a laboratory surrogate outcome measure (primary outcome: percentage change in endogenous thrombin potential) to assess the mechanism of action of the interventions in these patients. In addition, only few high-risk patients with triple positivity were included.

Elsebaie et al performed a subgroup meta-analysis on patients with APS and venous thrombosis which included eight studies. The rate of recurrence of VTE in 233 patients with APS receiving a DOAC was not higher in comparison to 244 patients on warfarin or heparin. However, only a minority of the analyzed patients were high-risk patients with triple positivity. ${ }^{16}$

These data support the fact that low-risk APL patients with sole venous thrombosis and no vascular risk factors could profit from the use of DOACs. DOACs offer a reliable anticoagulation especially in patients with a first venous thrombosis and low or moderate risk profile APL without LA with the advantage that there is no need for laboratory monitoring.

Table 3 Secondary prevention after venous thromboembolism

\begin{tabular}{|c|c|c|c|}
\hline Clinical and laboratory findings & Therapy & Duration of therapy & $\begin{array}{l}\text { Additional therapy (to prevent } \\
\text { occurrence of arterial thrombosis) }\end{array}$ \\
\hline $\begin{array}{l}\text { First VTE and high-risk APL profile } \\
\text { (triple positivity or positive LA) }\end{array}$ & VKA (INR: $2-3$ ) & As long as APL persist ${ }^{a}$ & Minimize vascular risk factors ${ }^{b}$ \\
\hline $\begin{array}{l}\text { First VTE and low-to-moderate } \\
\text { risk APL profile (negative LA, } \\
\text { ACA, and/or } \beta 2 \text { CPIA) }\end{array}$ & VKA (INR: 2-3) or DOAC & As long as APL persist ${ }^{\mathrm{a}}$ & Minimize vascular risk factors ${ }^{b}$ \\
\hline Recurrent VTE while on DOAC & VKA (INR: 2-3) & As long as APL persist ${ }^{\mathrm{a}}$ & Minimize vascular risk factors ${ }^{\mathrm{b}}$ \\
\hline Recurrent VTE while on VKA & $\begin{array}{l}\text { VKA (INR: } 3-4) \\
\text { if recurrence occurred } \\
\text { despite an INR of } 2-3^{c}\end{array}$ & As long as APL persist ${ }^{a}$ & \\
\hline
\end{tabular}

Abbreviations: $\beta 2$ CPIA, $\beta 2$-glycoprotein I antibodies; ACA, anticardiolipin antibodies; APL, antiphospholipid antibodies; DOAC, direct oral anticoagulant; INR, international normalized ratio; LA, lupus anticoagulant; VKA, vitamin K antagonist; VTE, venous thromboembolism. aLaboratory assessment of APL at least once per year.

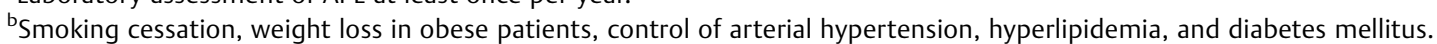

'Check for APL interfering with INR measurement; perform chromogenic factor-X assay and compare with INR measurement. 
Additionally, DOAC can be considered for patients who have difficulty to achieve a target INR of 2 to 3 despite compliance or who have contraindications to VKAs ${ }^{11}$ (see - Table 3 ).

Recently a meta-analysis of 47 studies corresponding to 447 patients treated with DOACs has been published. ${ }^{17}$ Patients treated with three commercially available DOACs were analyzed: rivaroxaban $(n=290)$, dabigatran etexilate $(n=144)$, and apixaban $(n=13)$. A total of 73 out of 447 patients $(16 \%)$ experienced a recurrent thrombotic event while on DOACs with a mean duration until thrombosis of 12.5 months. Rates of recurrent thromboses were 16.9 and $15 \%$ in APS patients receiving either anti-Xa inhibitors or dabigatran, respectively. Triple positivity was associated with a fourfold increased risk of recurrent thrombosis (56 vs. $23 \%$; odds ratio $[\mathrm{OR}]=4.3[95 \%$ confidence interval, $\mathrm{CI}$ : 2.3-7.7], $p<0.0001$ ) as well as a higher number of clinical criteria for APS classification. In patients treated with anti-Xa inhibitors, history of arterial thrombosis was associated with a higher risk of recurrent thrombosis ( 32 vs. $14 \%$; OR $=2.8$ [95\% CI: 1.4-5.7], $p=0.006)$.

This is supported by the results of the randomized openlabel multicenter noninferiority TRAPS study (Trial on Rivaroxaban in Antiphospholipid Syndrome). ${ }^{8}$ Rivaroxaban, $20 \mathrm{mg}$ once daily (15 mg once daily based on kidney function) was compared with warfarin (INR target 2.5) for the prevention of thromboembolic events, major bleeding, and vascular death in patients with APS. Only high-risk patients with triple positivity were included in the study. The trial was terminated prematurely after the enrollment of 120 patients (59 randomized to rivaroxaban and 61 to warfarin) because of an excess of events among patients in the rivaroxaban arm. Mean follow-up was 569 days. There were 11 (19\%) events in the rivaroxaban group, and 2 (3\%) events in the warfarin group. Thromboembolic events occurred in seven (12\%) patients randomized to rivaroxaban (four ischemic stroke and three myocardial infarction [MI]), whereas no event was recorded in those randomized to warfarin. Major bleeding occurred in six patients: four (7\%) in the rivaroxaban group and two (3\%) in the warfarin group. No death was reported. According to this trial, DOACs may be inferior to warfarin in high-risk (triple-positive) APL patients due to an increase of arterial events. It is important to note that lowrisk APL patients were excluded from the study and that no increase of VTE was observed.

Following the results of the TRAPS trial, the Pharmacovigilance Risk Assessment Committee (PRAC) of the European Medicines Agency (EMA) published a new product information wording for the DOACs apixaban, dabigatran etexilate, edoxaban, and rivaroxaban ${ }^{18}$ with special warnings and precautions for patients with APS on May $92019^{19}$ (https://www.ema. europa.eu/en/documents/other/new-product-informationwording-extracts-prac-recommendations-signals-adopted-811-april-2019-prac_en.pdf).

DOACs, including rivaroxaban, apixaban, edoxaban, and dabigatran etexilate, are not recommended for patients with a history of thrombosis who are diagnosed with APS. In particular, for patients that are triple positive (for LA, ACAs, and anti-B2GPIA), treatment with DOACs could be associated with increased rates of recurrent thrombotic events compared with VKA therapy.

In addition, on May 23, 2019 the German Federal Institute for Drugs and Medical Devices recommended for patients with APS, who are currently treated with a DOAC for prevention of thromboembolic events, to assess whether the continuation of this treatment is adequate and to consider switching to VKA, particularly in high-risk patients. $^{19}$

Therefore, in triple-positive patients or patients with LAs and venous thrombosis, VKAs remain the anticoagulants of choice and DOACs should be avoided in these special patient populations with APS ${ }^{20}$ (see - Table 3 ).

The ASTRO-APS study is currently testing apixaban for the secondary prevention of thrombosis among patients with APS (http://clinicaltrials.gov;NCT02295475). The study protocol was modified after the results of the TRAPS study had been published: patients with prior arterial thrombosis were excluded from enrollment and an increase of the dose of apixaban to $5 \mathrm{mg}$ twice daily instead of $2.5 \mathrm{mg}$ twice daily was performed. ${ }^{21}$ Results are not yet available but may change the above-mentioned recommendations.

\section{Chronic Thromboembolic Pulmonary Hypertension}

A possible complication of APL patients with PE is chronic thromboembolic pulmonary hypertension (CTEPH). This complication occurs in 1 to $5 \%$ of patients with PAPS and 0.5 to $14 \%$ of patients with SAPS. Recently 297 patients with CTEPH were retrospectively analyzed. Twenty-three patients (7.7\%) were positive for laboratory tests exploring APL antibodies. Among them, 17 patients (74\%) had a triple-positive APL profile. When compared with the APS-negative group, APS patients were significantly younger $(30.0 \pm 11.1$ vs. $55.6 \pm 12.9$ years, $p<0.0001)$, had more frequently a history of PE (95.6 vs. $65.7 \%, p=0.003$ ), and had more frequently associated autoimmune disease ( 43.5 vs. $2.9 \%, p<0.0001) .{ }^{22}$ The gold standard treatment for patients with CTEPH is curative pulmonary endarterectomy (PEA) combined with lifelong anticoagulant therapy. For patients with recurrent CTEPH after PEA riociguat, a guanylate cyclase stimulator is the only medicine available to this day to treat pulmonary arterial hypertension and inoperable persistent or recurrent CTEPH as it has been shown to improve exercise capacity. An emerging treatment strategy for persistent or inoperable CTEPH is balloon pulmonary angioplasty. This should be performed in CTEPH expert centers. ${ }^{23-25}$ As CTEPH is a potentially life-threatening disease, in patients with suspected CTEPH, a ventilation/perfusion or perfusion lung scan should be performed and they should be referred to CTEPH expert centers.

\section{Secondary Prevention of Arterial Thrombosis in Patients with APS}

Arterial complications are frequently observed in APS patients. These include ischemic stroke, transient ischemic 
attack (TIA), and acute MI often at a young age. APS is encountered in $17 \%$ of patients younger than 50 years diagnosed with $\mathrm{MI}$ and in $11 \%$ of young patients presenting with stroke or TIA. MI and positive APL have more frequently been reported in women. ${ }^{1,26}$ Arterial thrombosis of the upper and lower extremities occurs less frequently.

In 30 to $77 \%$ of patients with primary or SAPS, heart valve vegetations are present. These can cause cardiac emboli and subsequently cerebral ischemic attacks. Therefore, patients with APS should be initially screened with a transthoracic echocardiogram, whereas patients with APS and previous thrombosis, mainly arterial, should be routinely screened with transesophageal echocardiography. ${ }^{27,28}$

Currently the mainstay treatments of patients with arterial thromboses are VKAs with or without antiplatelet therapy (APT).The combined use should be independently validated for each patient according to the presence of vascular risk factors and the patient's bleeding risk. Using the aGAPSS score might help to detect patients who have a higher risk of recurrent thrombosis. The target INR should be between 2 and 3, although in some cases as in patients with recurrent arterial thrombosis despite an INR within the therapeutic range the INR should be kept above 3 and additional APT is recommended (see - Table 4).The use of hydroxychloroquine in addition to anticoagulation could be considered in patients with recurrent thrombotic events although there are no data available in patients with primary APS.

The TRAPS study compared rivaroxaban to warfarin in patients with triple-positive APL. ${ }^{8}$ The study was terminated early because patients on rivaroxaban showed significantly higher rates of occurrence of arterial events compared with warfarin-treated patients. Therefore, the use of DOAC is contraindicated in APS patients with previous arterial thrombosis or triple-APL positivity. ${ }^{19}$

In patients with stroke and a moderate or high-risk APL profile, the addition of low-dose aspirin (75-100 mg) should be considered. In older patients with low-risk APL profile and a high bleeding risk, low-dose aspirin alone may be considered. ${ }^{7}$
Patients with intracardial thrombosis need intensive anticoagulation (recommended INR: 3-4) and cardiosurgical consultation. $^{27}$

It is important to minimize all vascular risk factors in these patients: use of statins for correction of dyslipidemia, optimal treatment of diabetes mellitus, weight loss in the case of obesity, regular sports, smoking cessation, and control of hypertension are obligatory. Therefore, patient's counseling and education is very important.

Studies on alternative treatments, e.g., on the use of dual APT in APS patients with arterial events are limited. In a recent retrospective monocentric cohort study, 90 APL-positive patients with a first arterial thrombosis were divided into four therapeutic groups: 13 patients on warfarin monotherapy, 21 patients on warfarin combined with APT, 41 patients on antiplatelet monotherapy, and 15 patients on dual APT (DAPT). ${ }^{29}$ There were no differences in bleeding complications among the four groups. During DAPT recurrence rates of thrombotic events were equal to those of warfarin combined with APT. The most common thrombotic events were cerebral infarction, reported in 29 patients (32.2\%), and deep vein thrombosis, reported in 3 patients (3.3\%). Unexpectedly, patients treated with warfarin alone had a significantly higher risk of recurrence of cerebral infarction compared with DAPT. These differences in efficacy may be attributable to the constitutional difference in arterial thrombi initiated as platelet aggregates and venous thrombi consisting mainly of fibrin. ${ }^{30}$ Therefore, DAPT might be considered as an effective and safe alternative option for the prophylaxis of recurrent arterial thrombosis in APS but larger randomized prospective multicenter studies are needed to confirm these results.

\section{Catastrophic APS}

CAPS is a rare complication of APS (less than $1 \%$ of APS patients), which clinically presents as a "thrombotic storm," the development of excessive thrombosis at multiple sites, usually affecting small vessels and leading to multiorgan dysfunction and organ failure. ${ }^{1}$ According to a consensus statement, diagnosis is made when the following criteria are

Table 4 Secondary prevention after arterial thrombosis

\begin{tabular}{|c|c|c|c|}
\hline Clinical and laboratory findings & Therapy & $\begin{array}{l}\text { Duration of } \\
\text { therapy }\end{array}$ & Additional therapy \\
\hline $\begin{array}{l}\text { Arterial thrombosis and APL (moderate } \\
\text { and high risk) }\end{array}$ & $\begin{array}{l}\text { VKA (INR: } 2-3 \text { ) additional } \\
\text { low-dose aspirin } \\
\text { (75-100 } \mathrm{mg} \text { ) can be considered }\end{array}$ & $\begin{array}{l}\text { As long as APL } \\
\text { persist }^{\mathrm{a}}\end{array}$ & $\begin{array}{l}\text { Statins/minimize vascular } \\
\text { risk factors }{ }^{\mathrm{b}}\end{array}$ \\
\hline $\begin{array}{l}\text { Arterial thrombosis and low risk APL profile } \\
\text { or patients with high bleeding risk }\end{array}$ & $\begin{array}{l}\text { Consider low-dose aspirin } \\
(75-100 \mathrm{mg}) \text { alone }\end{array}$ & $\begin{array}{l}\text { As long as APL } \\
\text { persist }^{\mathrm{a}}\end{array}$ & $\begin{array}{l}\text { Statins/minimize vascular } \\
\text { risk factors }{ }^{\mathrm{b}}\end{array}$ \\
\hline $\mathrm{APL}$ and recurrent arterial thrombosis ${ }^{\mathrm{c}}$ & $\begin{array}{l}\text { VKA (INR: } 2-3 \text { ) and platelet } \\
\text { inhibitors, i.e., ADP receptor } \\
\text { inhibitor or VKA (INR: } 3-4 \text { ) }\end{array}$ & $\begin{array}{l}\text { As long as APL } \\
\text { persist }^{\mathrm{a}}\end{array}$ & $\begin{array}{l}\text { Statins/minimize vascular } \\
\text { risk factors } \\
\text { Consider } \\
\text { hydroxychloroquine }\end{array}$ \\
\hline
\end{tabular}

Abbreviations: APL, antiphospholipid antibodies; INR, international normalized ratio; VKA, vitamin K antagonist.

a Laboratory assessment of APL at least once per year.

bSmoking cessation, weight loss in obese patients, control of arterial hypertension, hyperlipidemia, and diabetes mellitus.

${ }^{c}$ Check for APL interfering with INR measurement; perform chromogenic factor-X assay and compare with INR measurement. 
present: (1) thrombosis affecting three or more organs or tissues, (2) presence of APL, (3) fast presentation of symptoms in less than 1 week, and (4) histopathological confirmation of microvascular thrombosis. ${ }^{4}$ It can be triggered by infections, recent major surgery, trauma, certain drugs, and withdrawal from anticoagulation, and has a very high mortality (higher than $50 \%$ in untreated patients). Multimodal treatment is therefore essential and necessary to save lives. ${ }^{1,5,31}$

A recent single-center report evaluated the clinical significance of laboratory findings in patients with CAPS as well as the effects of a well-defined treatment protocol in 14 consecutive cases. $^{32}$ Thirteen patients were consecutively treated and monitored between 1986 and 2017. APL characteristics of the patients were compared with those of 64 matched controls (45 APS patients and 19 APL carriers) who did not develop CAPS during the same mean follow-up period (12 years \pm 9.9 standard deviation). Triple-APL positivity (IgG/IgM anticardiolipin + IgG/IgM anti-B2GPIA + LAs) significantly prevailed in the CAPS patients with respect to the controls $(p=0.003)$. Early use of a defined treatment protocol based on triple therapy (anticoagulation + plasma exchange + steroids) either or not associated with intravenous immunoglobulin infusion was associated with recovery in all CAPS patients. Plasma exchange should start within 12 hours of onset ${ }^{33}$ especially if schistocytes are present.

Rituximab, an immunomodulatory treatment (anti-CD 20 antibody), has also been used in patients with CAPS refractory to standard treatment in combination with the previous reported treatment modalities. ${ }^{1}$ In addition to conventional treatment, inhibition of the complement by eculizumab has been reported in some refractory cases with successful inhibition of organ damage induced by APL. ${ }^{34}$

\section{Conflict of Interest}

Edelgard Lindhoff-Last has received lecture honoraria and advisory fees from Bayer AG, Boehringer Ingelheim, Bristol-Myers Squibb/Pfizer, Daiichi-Sankyo and Werfen and institutional research support from Bayer AG, BristolMyers Squibb/Pfizer, Daiichi-Sankyo and Werfen.

\section{References}

1 Linnemann B. Antiphospholipid syndrome - an update. Vasa 2018; 47(06):451-464

2 Miyakis S, Lockshin MD, Atsumi T, et al. International consensus statement on an update of the classification criteria for definite antiphospholipid syndrome (APS). J Thromb Haemost 2006;4 (02):295-306

3 Keeling D, Mackie I, Moore GW, Greer IA, Greaves M; British Committee for Standards in Haematology. Guidelines on the investigation and management of antiphospholipid syndrome. Br J Haematol 2012;157(01):47-58

4 Asherson RA, Cervera R, de Groot PG, et al; Catastrophic Antiphospholipid Syndrome Registry Project Group. Catastrophic antiphospholipid syndrome: international consensus statement on classification criteria and treatment guidelines. Lupus 2003;12 (07):530-534

5 Garcia D, Erkan D. Diagnosis and management of the antiphospholipid syndrome. N Engl J Med 2018;379(13):1290

6 Ząbczyk M, Kopytek M, Natorska J, Undas A. The effect of DOACStop on lupus anticoagulant testing in plasma samples of venous thromboembolism patients receiving direct oral anticoagulants. Clin Chem Lab Med 2019;57(09):1374-1381

7 Garcia D, Erkan D. Diagnosis and management of the antiphospholipid syndrome. N Engl J Med 2018;378(21):2010-2021

8 Pengo V, Denas G, Zoppellaro G, et al. Rivaroxaban vs warfarin in high-risk patients with antiphospholipid syndrome. Blood 2018; 132(13):1365-1371

9 Pengo V, Ruffatti A, Legnani C, et al. Clinical course of high-risk patients diagnosed with antiphospholipid syndrome. J Thromb Haemost 2010;8(02):237-242

10 Galli M, Luciani D, Bertolini G, Barbui T. Lupus anticoagulants are stronger risk factors for thrombosis than anticardiolipin antibodies in the antiphospholipid syndrome: a systematic review of the literature. Blood 2003;101(05):1827-1832

11 Gebhart J, Posch F, Koder S, et al. Increased mortality in patients with the lupus anticoagulant: the Vienna Lupus Anticoagulant and Thrombosis Study (LATS). Blood 2015;125(22):3477-3483

12 Radin M, Sciascia S, Erkan D, et al; APS ACTION. The adjusted global antiphospholipid syndrome score (aGAPSS) and the risk of recurrent thrombosis: Results from the APS ACTION cohort. Semin Arthritis Rheum 2019;49(03):464-468

13 Cohen $\mathrm{H}$, Efthymiou M, Isenberg DA. Use of direct oral anticoagulants in antiphospholipid syndrome. J Thromb Haemost 2018;16(06):1028-1039

14 Isert M, Miesbach W, Schüttfort G, et al. Monitoring anticoagulant therapy with vitamin $\mathrm{K}$ antagonists in patients with antiphospholipid syndrome. Ann Hematol 2015;94(08):12911299

15 Cohen H, Hunt BJ, Efthymiou M, et al; RAPS trial investigators. Rivaroxaban versus warfarin to treat patients with thrombotic antiphospholipid syndrome, with or without systemic lupus erythematosus (RAPS): a randomised, controlled, open-label, phase 2/3, non-inferiority trial. Lancet Haematol 2016;3(09): e426-e436

16 Elsebaie MAT, van Es N, Langston A, Büller HR, Gaddh M. Direct oral anticoagulants in patients with venous thromboembolism and thrombophilia: a systematic review and meta-analysis. J Thromb Haemost 2019;17(04):645-656

17 Dufrost V, Risse J, Reshetnyak T, et al. Increased risk of thrombosis in antiphospholipid syndrome patients treated with direct oral anticoagulants. Results from an international patient-level data meta-analysis. Autoimmun Rev 2018;17(10):1011-1021

18 Pharmacovigilance Risk Assessment Committee of the European Medicines Agency. New product information wording: Directacting oral anticoagulants (DOACs): apixaban; dabigatran etexilate; edoxaban; rivaroxaban - Recurrent thrombosis in patients with antiphospholipid syndrome (EPITT no 19320). Available at: https://www.ema.europa.eu/en/documents/other/new-productinformation-wording-extracts-prac-recommendations-signalsadopted-8-11-april-2019-prac_en.pdf2019

19 Bauersachs R, Schellong S, Stücker M, et al. Therapie des Antiphospholipid-Syndroms (APS) mit DOAKs. Haemostaseologie 2019;39(03):298-300

20 Tektonidou MG, Andreoli L, Limper M, et al. EULAR recommendations for the management of antiphospholipid syndrome in adults. Ann Rheum Dis 2019;78(10):1296-1304

21 Woller SC, Stevens SM, Kaplan DA, T Rondina M. . Protocol modification of apixaban for the secondary prevention of thrombosis among patients with antiphospholipid syndrome study. Clin Appl Thromb Hemost 2018;24(01):192

22 Jiang $\mathrm{X}$, Du Y, Cheng CY, et al. antiphospholipid syndrome in chronic thromboembolic pulmonary hypertension: a well-defined subgroup of patients. Thromb Haemost 2019;119(09): 1403-1408

23 Wang W, Wen L, Song Z, Shi W, Wang K, Huang W. Balloon pulmonary angioplasty vs riociguat in patients with inoperable chronic thromboembolic pulmonary hypertension: a systematic review and meta-analysis. Clin Cardiol 2019;42(08):741-752 
24 Simonneau G, D'Armini AM, Ghofrani HA, et al. Riociguat for the treatment of chronic thromboembolic pulmonary hypertension: a long-term extension study (CHEST-2). Eur Respir J 2015;45(05): 1293-1302

25 Galiè N, Humbert M, Vachiery JL, et al; ESC Scientific Document Group. 2015 ESC/ERS Guidelines for the diagnosis and treatment of pulmonary hypertension: the Joint Task Force for the Diagnosis and Treatment of Pulmonary Hypertension of the European Society of Cardiology (ESC) and the European Respiratory Society (ERS): endorsed by: Association for European Paediatric and Congenital Cardiology (AEPC), International Society for Heart and Lung Transplantation (ISHLT). Eur Heart J 2016;37(01):67-119

26 Cervera R, Piette JC, Font J, et al; Euro-Phospholipid Project Group. Antiphospholipid syndrome: clinical and immunologic manifestations and patterns of disease expression in a cohort of 1,000 patients. Arthritis Rheum 2002;46(04):1019-1027

27 Kolitz T, Shiber S, Sharabi I, Winder A, Zandman-Goddard G. Cardiac manifestations of antiphospholipid syndrome with focus on its primary form. Front Immunol 2019;10:941

28 Arnautovic JZ, Yamasaki H, Rosman HS. Multiple embolic strokes as a result of Libman-Sacks endocarditis associated with lupus and secondary antiphospholipid antibody syndrome: a case report. Eur Heart J Case Rep 2018;2(03):yty094

29 Ohnishi N, Fujieda Y, Hisada R, et al. Efficacy of dual antiplatelet therapy for preventing recurrence of arterial thrombosis in patients with antiphospholipid syndrome. Rheumatology (Oxford) 2019;58 (06):969-974

30 Mackman N. Triggers, targets and treatments for thrombosis. Nature 2008;451(7181):914-918

31 Espinosa G, Cervera R, Asherson RA. Catastrophic antiphospholipid syndrome and sepsis. A common link? J Rheumatol 2007;34 (05):923-926

32 Ruffatti A, De Silvestro G, Marson P, et al. Catastrophic antiphospholipid syndrome: lessons from 14 cases successfully treated in a single center. A narrative report. J Autoimmun 2018;93:124-130

33 Kazzaz NM, McCune WJ, Knight JS. Treatment of catastrophic antiphospholipid syndrome. Curr Opin Rheumatol 2016;28(03): 218-227

34 Tinti MG, Carnevale V, Inglese M, et al. Eculizumab in refractory catastrophic antiphospholipid syndrome: a case report and systematic review of the literature. Clin Exp Med 2019;19(03):281-288 